\title{
PVANET-HOUGH: DETECTION AND LOCATION OF CENTER PIVOT IRRIGATION SYSTEMS FROM SENTINEL-2 IMAGES
}

\author{
J. W. Tang ${ }^{1,2}$, D. Arvor $^{3}$, T. Corpetti ${ }^{3}$, P. Tang ${ }^{1, *}$ \\ ${ }^{1}$ Aerospace Information Research Institute, Chinese Academy of Sciences, Beijing, China - (tangjw, tangping)@aircas.ac.cn \\ ${ }^{2}$ School of Electronic, Electrical and Communication Engineering, University of Chinese Academy of Sciences, Beijing, China \\ ${ }^{3}$ CNRS, UMR 6554 LETG, Rennes, France - (damien.arvor, thomas.corpetti)@univ-rennes2.fr
}

\author{
Commission III, WG III/10
} KEY WORDS: Center Pivot Irrigation Systems, Object Detection, Deep Learning, Convolutional Neural Network, Hough
Transform

\begin{abstract}
:
Irrigation systems play an important role in agriculture. As being labor-saving and water consumption efficient, center pivot irrigation systems are popular in many countries. Monitoring the distribution of center pivot irrigation systems can provide important information for agriculture production, water consumption and land use. Deep learning has become an effective method for image classification and object detection. In this paper, a new method to detect the precise shape of center pivot irrigation systems, PVANET-Hough, is proposed. The proposed method combines a lightweight real-time object detection network PVANET based on deep learning and accurate shape detection Hough transform to detect and accurately locate center pivot irrigation systems. The method proposed in this paper does not need any preprocessing, PVANET is lightweight and fast, Hough transform can accurately detect the shape of center pivot irrigation systems, and reduce the false alarms of PVANET at the mean time. Experiments with the Sentinel-2 images in Mato Grosso demonstrated the effectiveness of the proposed method.
\end{abstract}

\section{INTRODUCTION}

Irrigation systems have import impact on the quality of agriculture production. The use of irrigation systems is an important part of the modernization of agriculture production and intensification management (Arvor et al., 2012). Mapping the distribution of irrigation systems helps in the understanding of agriculture production and consumption of water resources. Center pivot irrigation system is popular in countries like USA, Brazil and Israel. Center pivot irrigation systems are easy to recognize in satellite images, because the crops fields approximate to circular shape. Monitoring with satellite images can get the locations and distributions of center pivot irrigation systems, which is import information about ongoing development trends in agriculture.

Detecting the distribution of center pivot irrigation systems in satellite images is cheap. Center pivot irrigation systems can be mapped by detecting circles in Satellite images, which is how humans interpret center pivot irrigation systems in satellite images. The basic method to automatically detect circles is Hough transform (Duda, Hart, 1971). Hough transform first convert each points in image space into parameter space, then search for objects by voting. However, using Hough transform directly to detect circular objects in satellite images has the drawbacks of low precision, long computation time and large data storage (Chiu et al., 2010).

Recently, Deep learning has become an effective solution for the tasks of image classification (Huang et al., 2016) and object detection (Redmon, Farhadi, 2018). Especially, CNN (convolution neural network), has been applied to detect all kinds of objects in images. Zhang (Zhang et al., 2018) explored to use $\mathrm{CNN}$ to detect center pivot irrigation systems in TM images, the proposed method consists of three steps. The first step is preprocessing, which is masking the images with CDL (Landsat data and Crop Data Layer) to rule out noncropland areas. The second step is sliding a fixed sized window over the image, then fed the window image into LeNet-based, AlexNet-based or VGGNet-based networks to determine whether the window is a center pivot irrigation system or not. The third step is locating center pivot irrigation systems using a variance based approach. The proposed method has a high accuracy, but also has a lot of redundant computation, the process is very slow.

Actually, as the neighborhood information always participating, CNN based methods have an innate drawback of not being able to detect the shapes precisely, CNN learns more about the implicit representations of shape and texture (Milletari et al., 2016), the prediction of which is more relatively shape and location. Therefore other means are needed to detect the location of objects accurately, which is why (Zhang et al., 2018) uses variance based approach to locate the center pivot irrigation systems and requires preprocessing to reduce false alarms.

With a prior Knowledge of the shape, a new method to detect the accurate shape of center irrigation systems, PVANETHough, is proposed in this paper. The proposed method combines the deep learning method of object detection and accurate shape detection Hough transform, which integrates a lightweight real-time object detection network PVANET (Kim et al., 2016) and accurate shape detecting Hough voting to

\footnotetext{
* Corresponding author
} 
detect and accurately locate center pivot irrigation systems. The method proposed in this paper does not need any preprocessing, PVANET is fast, Hough voting can accurately locate the center and shape of center pivot irrigation systems, and at the mean time reduce the false alarms of the results of PVANET as it cannot detect the shape precisely.

The rest of the paper is organized as follows. Description of experiment data is in Section 2. The PVANET-Hough method is described in Section 3. Experiment results and discussion is presented in Section 4. Section 5 concludes the work.

\section{DATA}

\subsection{Study Area}

The study area is located in the Brazilian state of Mato Grosso, in the southern Amazon as shown in Figure 1. Mato Grosso is located in the center-west of Brazil, third largest state by area, total area of which is 903357 square kilometers. Mato Grosso is a big state of agriculture, the biggest region for soybeans and corn, agriculture makes up $40.8 \%$ of the state's GDP. Detecting the center pivot irrigation systems in Mato Grosso is of great importance to analyze the process of agricultural intensification in the state.

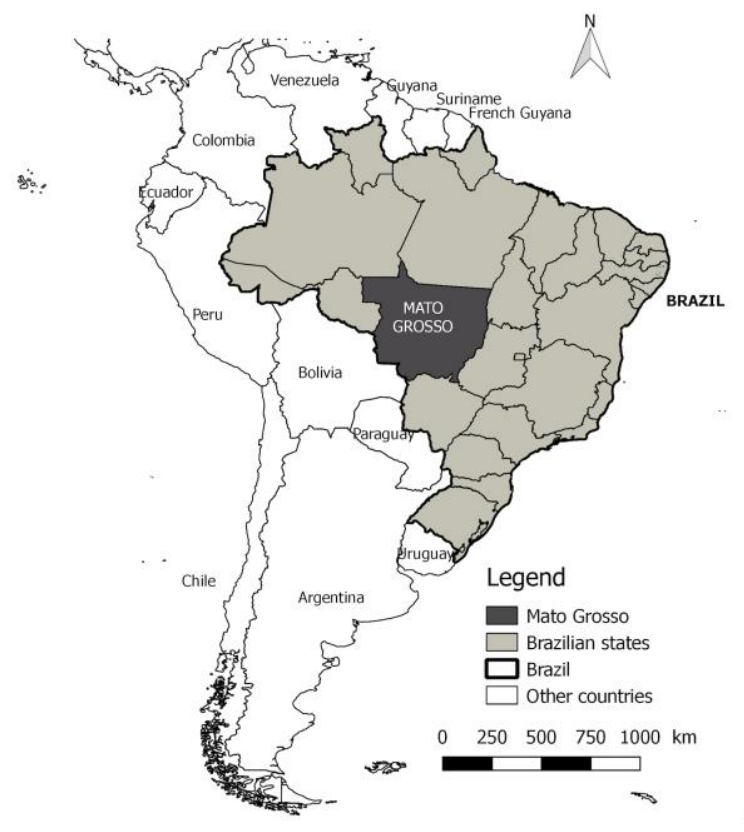

Figure 1. The location of Mato Grosso in Brazil

\subsection{Images}

The satellite images used in the study are sentinel-2 images. More precisely, TCI (True Color Image) images are used to detect the center pivot irrigation systems. The spatial resolution is 10 meters. The images used in the study cover three major Amazon watersheds in Mato Grosso (Juruena, Teles Pires and Xingu river), It is around $750,000 \mathrm{~km}^{2}$ and $2 / 3$ of Mato Grosso, 77 images in total, acquired from June to August 2013. The size of every image is $10980 * 10980$ pixels. All the images are processed in the unified framework called PVANET-Hough to get the locations of the center pivot irrigation systems in the region.

\section{PVANET-HOUGH}

The proposed PVANET-Hough method consists of two parts: PVANET for detection of center pivot irrigation systems, Hough transform for accurate location of center pivot irrigation systems, as shown in Figure 2, which are described in the subsections.

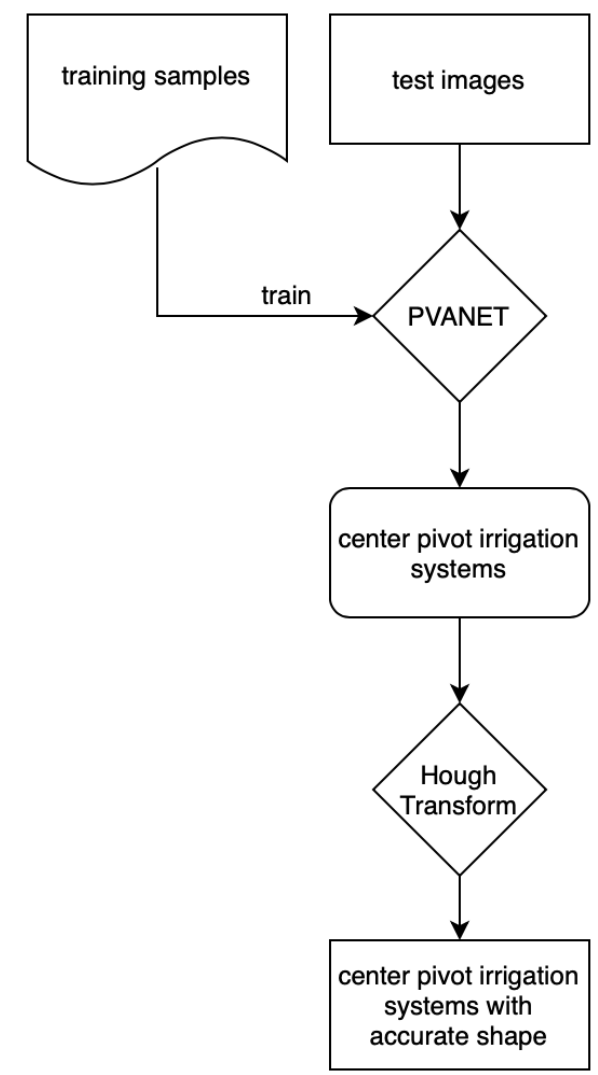

Figure 2. Flowchart of the PVANET-Hough method

\subsection{PVANET for Detection of Center Pivot Irrigation Systems}

3.1.1 The Architecture of PVANET: PVANET (Performa nce vs Accuracy Network) is the name for deep but lightweigh $\mathrm{t}$ neural networks for real-time object detection. This method ca $\mathrm{n}$ achieve real-time object detection performance without losing accuracy compared to the other state-of-the-art systems, such a s ResNet-101 (He et al., 2015).

The structure of PVANET detection network is shown in Figure 3. The pipeline of PVANET is the same as Faster R-CNN (Ren et al., 2015), which is "CNN feature extraction + region proposal + RoI classification", with redesign on the feature extraction part, adopting building blocks including concatenated C.ReLU (Shang et al., 2016), Inception (Szegedy et al., 2015) and HyperNet (Kong et al., 2016) to make the network thin and light, maximize the computational efficiency.

C.ReLU is illustrated in Figure 4. An interesting property of CNNs is observed, that in the early layers, the filters tend to form pairs. For every filter, there is another filter which is almost the opposite phase. Inspired by this observation, C.ReLU reduces the number of convolution channels by half, then concatenates the same outputs with negation, which is simply multiplies -1 to the output of convolution, leading to 2 


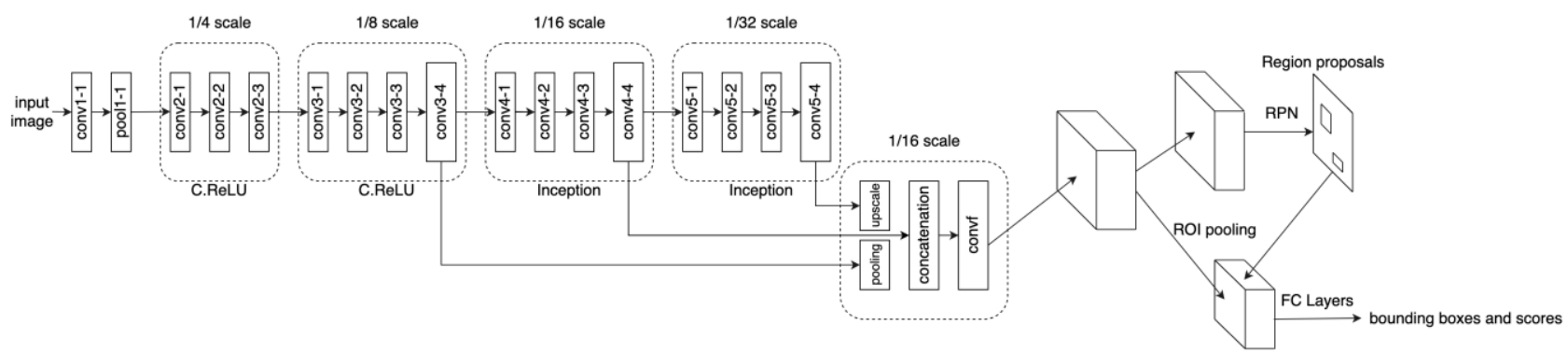

Figure 3. The structure of PVANET

times speed-up of the early stage without losing accuracy. Scaling and shifting after concatenation is appended to allow each channel's slope and activation threshold can be different from those of its opposite channel.

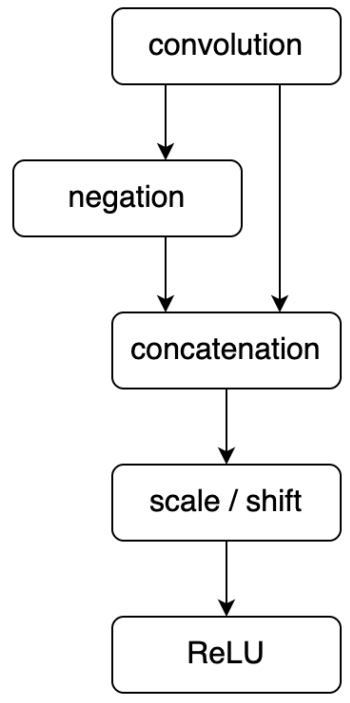

Figure 4. C.ReLU

Inception is one of the most cost-effective building block for capturing both small and large objects in an input image. To capture large objects, the receptive fields of the features of CNN should be large enough. On the other hand, to capture small objects, the receptive fields should be small enough to accurately localize them. As illustrated in Figure 5, Inception fulfills both requirements by congregating different sizes of kernels to the convolution layers. $1 \times 1$ convolution plays an import role by preserving the receptive field of the previous layer, slowing down the growth of receptive fields so that small objects can be precisely captured. In the implementation of Inception in PVANET. $5 \times 5$ convolution is replaced with a sequence of two $3 \times 3$ convolutions

HyperNet: Multi-scale features are proven to be beneficial in many deep learning tasks (Kong et al., 2016; Bell et al., 2016). Combining shadow fine-grained details with deep highlyabstracted information in feature layers helps the following region proposal network and classification network to detect objects of different scales. PVANET combines the last layer and two intermediate layers with scales of $2 \mathrm{x}$ and $4 \mathrm{x}$ of the last layer respectively. The middle size layer is chosen to be the reference layer and the down-scaled (pooling) $4 \mathrm{x}$-scaled layer and the up-scaled (linear interpolation) last layer are concatenated.

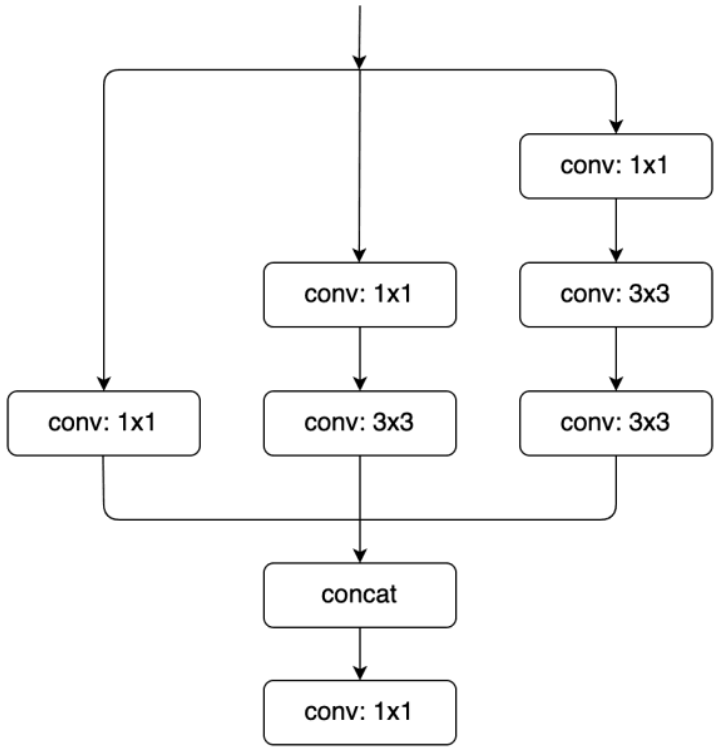

Figure 5. Inception

3.1.2 Training and Validation of PVANET: We fine-tune the pre-trained model of PVANET from ILSVRC2012 training images for 1000-class image classification. To fine-tune PVANET, a set of images with center pivot irrigation systems are sampled to be training and validation samples. In this paper, images with the size of $500 * 500$ are randomly cropped from a sentinel-2 image of Mato Grosso gained in July of 2013, 448 images with center pivot irrigation systems are selected from the cropped images, with the center pivot irrigation systems annotated, are used for the training samples of PVANET. Examples of training samples are shown in Figure 6, both complete and incomplete center pivot irrigation systems are included in the training samples, randomly located in the images, $10 \%$ of the training samples are used for validation samples.

The initial learning rate is set to be 0.001 , when the loss is detected to be in a plateau, the learning rate will be decreased by a factor of 0.1 .

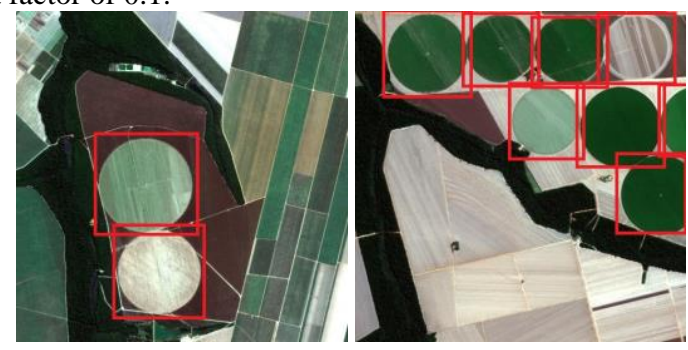

Figure 6. Examples of training samples 


\subsection{Accurate Location of Center Pivot Irrigation Systems by Hough Transform}

As CNN based object detection methods can only predict the location and shape of objects relatively, some objects with a shape similar to circle such as forest blocks may be mistakenly detected to be center pivot irrigation systems. Because the crops under center pivot irrigation systems have the unique shape of circle, we can detect these shapes to locate them and get the coordinates of the centers and radius. Hough transform is used in this paper. HT is highly reliable and adaptive to noise, transform, deformity and so on (Chuang et al., 2010). Hough transform calculates the maximum accumulated local results in a parameter space through voting algorithm. The collection of distinctive forms can be obtained. Therefore, the HT can be used to detect objects with particular shapes. We only detect circles here.

The Hough circle detection is to map the edge pixels from image space to $3 \mathrm{D}$ parameter space, then an arbitrary point on the circle will be transformed into a right circular cone in the 3D parameter space. All the image points on a circle whose cones will intersect at a single point in the $3 \mathrm{D}$ space. By searching a 3D Hough search space to find the centroid and radius of each circular object in an image space, this would mean far greater memory requirements and much slower speed. To solve these problems, researchers brought up some improved methods, 2-1 Hough Transform (21HT) is one of them, which is proposed in (Yuen et al., 2001). In the 21HT, to reduce the storage requirement, 2-D accumulator and a 1-D histogram are used to substitute 3D Hough search space.

This paper uses the $21 \mathrm{HT}$ to realize fast process. Firstly, the image is passed through an edge detection phase. Secondly, for every nonzero point in the edge image, the local gradient is considered. 2-D accumulator is used to accumulate votes along the normal of each edge point. 1-D histogram as a radius histogram is used to identify the radius of circles of the distance of each point from a candidate center. The detection of false peaks in the center finding stage can lead to significant computational cost for the second stage, especially if a low threshold is used to detect small circles. Since only a single 2-D accumulator and a 1-D histogram are used, the storage space required for the method is quite small. Moreover, according to the prior knowledge, the radius of a circular can be limited to the scale of (rmin, rmax), further improve the detecting speed. The 21HT run much faster and helps overcome the problem of the otherwise sparse population of $3 \mathrm{D}$ accumulator.

With the described method above, center pivot irrigations systems with the shape of circle can be accurately detected and located, as shown in Figure 7.

\section{RESULTS}

PVANET was implemented with Caffe. Training and testing were done in a machine with 8 cores, 16 GB RAM and NVIDIA GEFORCE GTX 1070. The training took 8 hours. We evaluate the results with the 77 images in Mato Grosso, the size of the image is $10980 * 10980$ pixels, the image is cropped into blocks of $500 * 500$ pixels with an overlap of 200 pixels between the neighborhood blocks, these blocks of images are fed into PVANET to detect the center pivot irrigation systems. After all the blocks of the image are detected, the duplicate detections between the blocks are removed to get the detections of the whole image. Hough transform is applied to the detections of PVANET to finally determine if the detection is a center pivot irrigation systems and get the accurate shape.

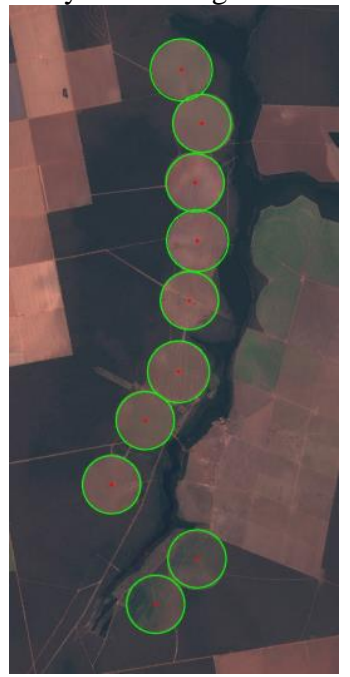

Figure 7. Center pivot irrigation systems accurately detected by Hough circle detection

PVANET and PVANET-Hough are compared in the experiment. Since Hough transform used in this paper is to exclude those PVANET mistakenly detected center pivot irrigation systems, so we mainly examine the ability of Hough transform to reduce the false detection rate. We use two quantitative indexes to evaluate the result: missed detection rate and false detection rate or precision and recall, since missed detection rate $=1$-recall and false detection rate $=1$-precision. Precision is defined as the number of correct detections over the number of correct detection plus the number of false alarms, which tells us how many of the detected center pivot irrigation systems are correct, while recall is defined as the number of correct detections over the number of ground truth, which tells us how many of the center pivot irrigation systems that should be detected are detected. The ground truth is manually identified. There are 641 center pivot irrigation systems in the images of Mato Grosso.

The result of PVANET is shown in Table 1, there are 752 detected candidates of center pivot irrigation systems by PVANET, 587 of the detection candidates are correct, 165 of the detection candidates are false, 54 center pivot irrigation systems are missed. The precision is $78.1 \%$, the recall is $91.6 \%$, the missed detection rate is $8.4 \%$ and the false detection rate is $21.9 \%$. Obviously, PVANET method has very high false detection rate. Figure 8 is some examples of the false detections of center pivot irrigation systems, it shows that a lot of forest patches with contour similar to circle and river bank are mistakenly detected by PVANET.

\begin{tabular}{|c|c|c|}
\hline Ground Truth & $\begin{array}{c}\text { Detected candidate } \\
\text { center pivots by } \\
\text { PVANET }\end{array}$ & $\begin{array}{c}\text { Correctly detected } \\
\text { center pivots by } \\
\text { PVANET }\end{array}$ \\
\hline 641 & 752 & 587 \\
\hline
\end{tabular}

Table 1. Results of PVANET 

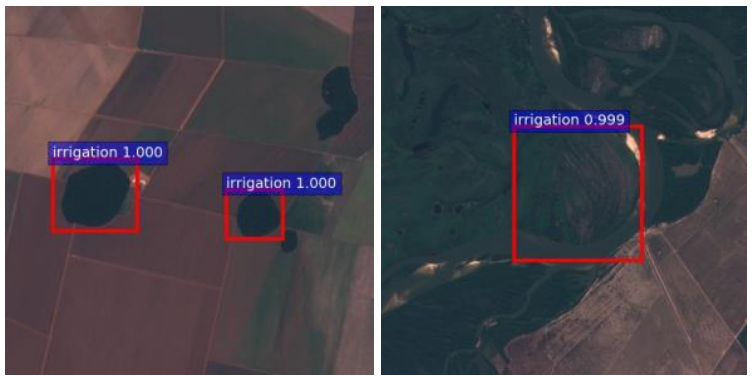

Figure 8. Examples of false detections by PVANET

The result of PVANET-Hough is shown in table 2, there are 662 detected candidate center pivot irrigation systems, 583 of the detection are correct. That means that Hough transform decreased the number of detected candidates from 752 to 662 so that, there are 79 false detections left. Examples of false detections by PVANET-Hough are shown in Figure 9. They are forest patches and circular farmland. We can notice that 583 detections are correct in all the candidate detections of PVANET-Hough, that means 58 center pivot irrigation systems are missed, 4 more are missed by the PVANET-Hough. Some missed center pivot irrigation systems by PVANET-Hough are shown in Figure 10. They are mainly incomplete center pivot irrigation systems at the border of images. The precision of PVANET-Hough is $88.1 \%$, the recall is $91 \%$, the missed detection rate is $9 \%$ and the false detection rate is $11.9 \%$, we can see the false detection of PVANET-Hough is decreased by a large percent, with just a very small decrease in the recall.

\begin{tabular}{|c|c|c|}
\hline Ground Truth & $\begin{array}{c}\text { Detected candidate } \\
\text { center pivots by } \\
\text { PVANET-Hough }\end{array}$ & $\begin{array}{c}\text { Correctly detected } \\
\text { center pivots by } \\
\text { PVANET-Hough }\end{array}$ \\
\hline 641 & 662 & 583 \\
\hline
\end{tabular}

Table 2. Results of PVANET-Hough
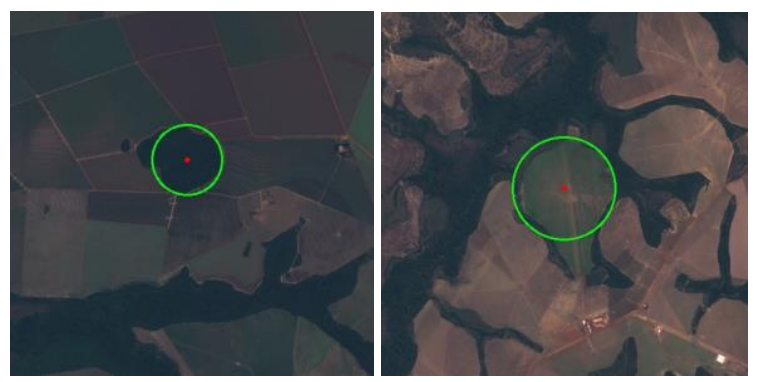

Figure 9. Examples of false detections by PVANET-Hough
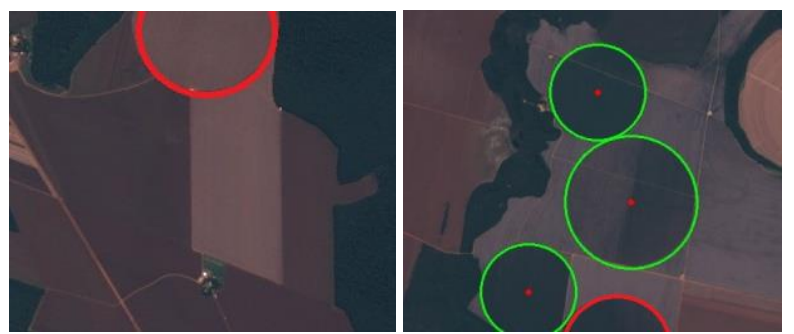

Figure 10. Missed detections of PVANET-Hough (red ones)

PVANET is not able to locate the shapes of center pivot irrigation systems accurately and there are many false alarms, as we can see from the result. Using Hough transform to the detections of PVANET can accurately locate the shapes of center pivot irrigation systems and reduce the false alarms of PVANET, which proves the effectiveness of the proposed method in this paper. Examples of the detection are shown in Fig 11.

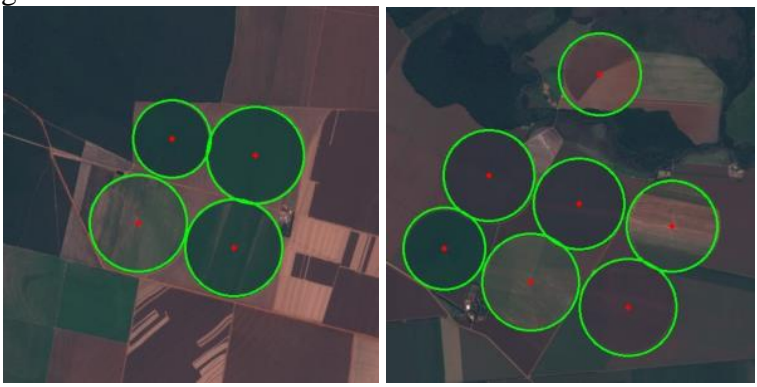

Figure 11. Examples of detections by PVANET-Hough

\section{CONCLUSION}

In this paper, PVANET-Hough, a method combining PVANET and Hough transform is proposed for the detection and accurate location of center pivot irrigation systems. PVANET is lightweight and fast, Hough transform can accurately locate the shapes of center pivot irrigation systems and meanwhile reducing the false alarm of CNN based method PVANET as it cannot precisely detect shapes. Experiment with the Sentinel-2 images in Mato Grosso proved the effectiveness of the proposed method in reducing the false detections by PVANET. In the future, we will do further research on how to reduce the miss detections by modifying PVANET-Hough and further improve the ability to reduce the false detections.

\section{ACKNOWLEDGEMENTS}

This work was supported by the Strategic Priority Research Program of the Chinese Academy of Sciences [Grant No. XDA19080301].

\section{REFERENCES}

Arvor, D., Meirelles, M., Dubreuil, V., Bégué, A., Shimabukuro, Y.E., 2012: Analyzing the agricultural transition in Mato Grosso, Brazil, using satellite-derived indices. Applied Geography, 32(2), 702-713. doi.org/10.1016/j.apgeog.2011.08.007.

Bell, S., Zitnick, C.L., Bala, K., Girshick, R., 2016: Insideoutside net: Detecting objects in context with skip pooling and recurrent neural networks. 2016 IEEE Conference on Computer Vision and Pattern Recognition (CVPR). doi.org/10.1109/cvpr.2016.314.

Chiu, S.H., Liaw, J.J., Lin, K.H., 2010: A Fast Randomized Hough Transform for Circle/Circular Arc Recognition. International Journal of Pattern Recognition and Artificial Intelligence, 24(03), 457-474. doi.org/10.1142/s0218001410007956.

Chuang, C.H., Lo, Y.C., Chang, C.C., Cheng, S.C., 2010: Multiple Object Motion Detection for Robust Image Stabilization Using Block-Based Hough Transform. 2010 Sixth International Conference on Intelligent Information Hiding and Multimedia Signal Processing. doi.org/10.1109/iihmsp.2010.158. 
Duda, R.O., Hart, P.E., 1972: Use of the Hough transformation to detect lines and curves in pictures. Communications of the ACM, 15(1). doi.org/10.1145/361237.361242.

He, K.M., Zhang, X.Y., Ren, S.Q., Sun, J., 2016: Deep Residual Learning for Image Recognition. 2016 IEEE Conference on Computer Vision and Pattern Recognition (CVPR). doi.org/10.1109/cvpr.2016.90.

Huang, G., Liu, Z., Maaten, L.V.D., Weinberger, K.Q., 2016: Densely Connected Convolutional Networks. Arxiv, arXiv: 1608.06993.

Kim, K.H., Hong, S.H., Roh, B.S., Cheon, Y.J., Park, M.J., 2016: PVANET: Deep but Lightweight Neural Networks for Real-time Object Detection. Arxiv, arXiv:1608.08021.

Kong, T., Yao, A.B., Chen, Y.R., Sun. F.C., 2016: HyperNet: Towards accurate region proposal generation and joint object detection. 2016 IEEE Conference on Computer Vision and Pattern Recognition (CVPR). doi.org/10.1109/cvpr.2016.98.

Milletari, F., Ahmadi, S.A., Kroll, C., Plate, A., Rozanski, V., Maiostre, J., Levin, J., Dietrich, O., Ertl-Wagner, B., Bötzel, K., Navab, N., 2016: Hough-CNN: Deep Learning for Segmentation of Deep Brain Regions in MRI and Ultrasound. Computer Vision and Image Understanding, 164, 92-102. doi.org/10.1016/j.cviu.2017.04.002.

Redmon, J., Farhadi A., 2018: YOLOv3: An Incremental Improvement. Arxiv, arXiv: 1804.02767.

Ren, S.Q., He, K.M., Girshick, R., Sun. J., 2015: Faster R-CNN: Towards real-time object detection with region proposal networks. In Advances in Neural Information Processing Systems (NIPS), 2015.

Shang, W.L., Sohn, K., Almeida, D., Lee, H.L., 2016: Understanding and Improving Convolutional Neural Networks via Concatenated Rectified Linear Units. ICML'16: Proceedings of the 33rd International Conference on International Conference on Machine Learning, 48, 2217-2225.

Szegedy, C., Liu, W., Jia, Q.Y., Sermanet, P., Reed, S., Anguelov, D., Erhan, D., Vanhoucke, V., Rabinovich A., 2015: Going deeper with convolutions. 2015 IEEE Conference on Computer Vision and Pattern Recognition (CVPR). doi.org/10.1109/cvpr.2015.7298594.

Yuen, H.K., Proncen, J., Illingworth, J., Kittler, J., 1990: Comparative Study of Hough Transform Methods for Circle Finding. Image and Vision Computing, 8(1), 71-77. doi.org/10.1016/0262-8856(90)90059-E.

Zhang, C.X, Yue, P., Di, L.P., Wu. Z.Y., 2018: Automatic Identification of Center Pivot Irrigation Systems from Landsat Images Using Convolutional Neural Networks. Agriculture, 8(10), 147. doi.org/10.3390/agriculture8100147. 